\title{
Linx
}

Revue des linguistes de l'université Paris X Nanterre

$54 \mid 2006$

La cause : approche pluridisciplinaire

\section{L'expression de la « cause » à travers le prisme de la préposition par}

\section{Badreddine Hamma}

\section{(2) OpenEdition}

\section{Journals}

Édition électronique

URL : http://journals.openedition.org/linx/505

DOI : $10.4000 /$ linx.505

ISSN : 2118-9692

\section{Éditeur}

Presses universitaires de Paris Nanterre

\section{Édition imprimée}

Date de publication : 1 juin 2006

Pagination : 73-90

ISSN : 0246-8743

\section{Référence électronique}

Badreddine Hamma, «L'expression de la « cause » à travers le prisme de la préposition par », Linx [En ligne], 54 | 2006, mis en ligne le 01 août 2007, consulté le 19 avril 2019. URL : http:// journals.openedition.org/linx/505; DOI : 10.4000/linx.505 


\title{
L'expression de la « cause » à travers le prisme de la préposition $p a r^{*}$
}

\author{
Badreddine HAMMA, Université de Paris $X$ - Nanterre \\ MoDyCo (UMR 7114)
}

\section{Introduction}

Parler d'une valeur "causale», indépendamment du co(n)texte dans lequel s'insère l'énoncé qui la véhicule, soulève de nombreuses difficultés d'analyse ; en effet, ce que l'on a coutume de regrouper traditionnellement sous l'étiquette «cause » ne renferme pas de valeurs homogènes. Cet aspect pluriel de la cause lui vient, notamment, du marqueur $(R)$ liant un premier segment de discours $X$ et un autre $Y$, ainsi que différentes unités lexicales mises en jeu dans un même énoncé : si on prend, par exemple, car, parce que et puisque (des marqueurs dits « intrinsèquement causaux $»^{1}$ ), en guise de $R$ dans la suite $P(R) Q$, on verra que l'on passe respectivement, selon les distributions permises, du cas où le locuteur donne un commentaire légitimant ou argumentant un comportement, un fait, etc. avec car (à ce propos, voir C. Normand,

\footnotetext{
* Je tiens à remercier les relecteurs anonymes pour leurs précieuses remarques, critiques et suggestions, qui m'ont été nécessaires pour affiner l'analyse dans la version finale de mon texte et qui m’ont incité, par ailleurs, à rendre plus explicites certaines de mes idées.

${ }^{1}$ Ici, nous ne prétendons pas caractériser de façon minutieuse les conjonctions car, parce que et puisque; mais nous admettons qu'elles expriment la «cause» de façon intrinsèque (mais avec des nuances propres à chaque unité) en ce sens que ces trois marqueurs ne sont pas répertoriés dans des emplois autres que ceux de la "cause » (ou l'un des sens qui lui sont contigus : «justification », « explication», « excuse », «prétexte», etc.) : généralement, ils ne sont pas employés dans des énoncés dont le sens global est l'« espace », le «temps », la «manière », etc., contrairement aux prépositions dont l'éventail d'emplois est assez large ( $c f . d e, p a r, a ̀, ~ e n, ~ e t c$.$) .$
} 
2002), à la justification d'un point de vue, d'un comportement, etc., généralement ignorés par l'interlocuteur - ou qui fait semblant de les ignorer -, avec parce que, à l'allusion à une cause évidente, déjà connue par l'interlocuteur, avec puisque - (pour une étude détaillée de ces trois marqueurs, voir S. Hamon 2005). Toutefois, quand $R$ est une préposition, par exemple, dans, de, par, pour, etc., la construction de la phrase véhiculant ce rapport dit de «cause» fait preuve d'un comportement davantage « erratique », comme le souligne P. Cadiot (1997 : 38). De fait, l'étiquette " préposition causale» devient elle-même problématique, parce que cela marginalise les autres emplois que connaissent ces marqueurs et revient ainsi à confondre le "signifié » propre de ces prépositions, (que l'on retrouve en principe dans toutes leurs manifestations discursives) avec leurs possibilités d'usage, qui relèvent d'un phénomène discursif (une affaire de $\operatorname{co}(\mathrm{n})$ texte). En d'autres termes, la critique que nous adressons à la tradition ne consiste pas à remettre en cause l'expression de la "cause » en soi, mais à l'imputer aux marqueurs. Nous admettons, de fait, que l'étiquette «expression de cause » est pertinente tant qu'elle n'est pas projetée sur la préposition elle-même ; il n'en reste pas moins que celle-ci y apporte sa contribution de façon sensible; elle permet de voir la valeur globale en question d'une certaine manière en fonction de son sens instructionnel.

D. Leeman (1990: 6), d'ailleurs, a pointé le revers de ce type d'approche, adopté dans les ouvrages usuels, et a fait remarquer que cette démarche est trompeuse du fait qu'elle entraîne des généralisations erronées; pour preuve, ce regroupement sous la même étiquette "prépositions exprimant la cause » ne permet pas de prévoir quelle préposition va avec quels noms ou quels verbes ; si l'on a aussi bien rougir de modestie ou rougir par modestie, on ne dit pas semblablement :

(1) Rougir (de+ ??par) bonte.

(2) Travailler (par $+{ }^{*}$ de) plaisir.

Si l'on peut construire également punir un élève par étourderie ou punir un élève pour étourderie, on n'a pas indifféremment :

(3) S'arrêter (pour+*par+* de) raisons de santé (D. Leeman, ibid.).

De même, la préposition dans n'est commutable avec aucune des précédentes dans :

(4) Il est mort (dans+ *par +*pour+*d') un accident de voiture (D. Leeman, 1990).

(5) (Dans+ *Par +*Pour+*De) son affolement, Max a oublié de fermer le gaz. (D. Leeman, 1998).

Mais on aurait bien Dans son affolement, Max a ému Léa et Par son affolement, Max a ému Léa ${ }^{2}$. Certes, dans, de, par et pour ici entrent dans des phrases exprimant une certaine notion de "cause", en (1-5), mais soumise à chaque fois à des contraintes linguistiques particulières. C'est pourquoi il faudrait, d'une part, souligner en quoi ces

\footnotetext{
2 Par son affolement, ici, s'apprête davantage à l'expression de la «manière » que celle de la «cause » (cf. infra $\$ 1.4$.$) .$
} 
prépositions se distinguent les unes des autres : la «cause » n'est pas exprimée de la même manière; d'autre part, préciser que la valeur «causale» n'est qu'un sens en emploi ; elle provient de l'interaction des éléments cotextuels mis en jeu dans l'énoncé, et, partant, elle est extrinsèque à la préposition en soi : par n'est plus, par exemple, censé véhiculer la cause dans partir par avion, sortir par la fenêtre, envoyer un message par fax, être écrit par Max, etc. Selon nous, cette préposition possède un signifié spécifique qui rend possible de telles interprétations et que l'on peut déceler dans toutes ses actualisations discursives, d'où la nécessité de déterminer cette spécificité. Nous nous focalisons, dans ce qui suit, sur le cas de par. Nous avons pu identifier deux types de construction pouvant contenir un sens «causal» où cette préposition laisse une empreinte sémantique importante : en par $N$ et en par ce que $P$. Ici, nous nous intéressons uniquement au premier type d'emploi ( $c f$. pour une étude détaillée des occurrences en par ce que P, B. Hamma, 2005).

\section{Identification des propriétés distributionnelles des occurrences en $\operatorname{par} N$}

Dans le but d'appréhender les spécificités de l'emploi « causal » où apparaît par, nous nous proposons d'examiner les principales propriétés linguistiques des $\mathrm{N}$ qui se combinent avec cette préposition dans la relation $(V)$ par $N$ ainsi que le reste des arguments de la phrase, en vue d'en établir le corrélat sémantique.

Considérons le corpus représentatif suivant ${ }^{3}$ :

(6) L'une est unie par un lien mère-fils à l'autre, le fils en avant de la mère et la guidant par nécessité plus que par amour.

(7) Ce sont les hommes, par jalousie, qui me dépeignent comme un monstre.

(8) Les misérables paysans l'accablaient d'injures, lui lançaient, à son passage, des pierres, des aufs pourris, et de viles matières que par décence je ne nomme pas.

(9) Je l'ai fait tout autant par vanité que par bonnêteté.

(10) Il n'a jamais dit de bien de qui que ce soit, par peur de se déprécier.

(11) Les curieux les laissèrent seuls, pas tellement par discrétion, mais parce que la chanson des fourchettes les appelait dans la grand-salle.

(12) Non point par ennui, mais par peur.

\footnotetext{
${ }^{3}$ Ce corpus a été élaboré à partir de la base de données de textes français Frantext. La principale requête que nous avons effectuée consiste à saisir, dans le premier champ de l'interface de la base, la préposition par, dans le second, une liste à choix des $\mathrm{N}$ que nous avons rassemblés à partir du corpus de D. Leeman (1998 : 197-206) et d'Y. Y. Mathieu (2002 : 145-157); dans le troisième champ, nous avons exclu les déterminants \&e $(\mathrm{g}=\mathrm{D})$, puis nous les avons intégrés, le tout avec un écart entre 0 et 3 mots, ce qui nous a donné 1006 occurrences. Faute de place, nous ne considérons, ici, que quelques occurrences représentatives.
} 
(13) Mais ce n'était point par vanité que Giulio se montrait faisant la leçon à ce malheureux célèbre, c'était par crainte, pour se laver du soupson de l'avoir jamais approuvé.

(14) Ils aiment à respecter, par bonté, et par modestie.

(15) Or, par bumilité, il préfere être tenu pour un ingrat.

(16) Je ne regarde pas par cruauté mais par pitié.

(17) Voici quelques-unes des définitions le plus fréquemment citées : touriste : voyageur qui ne parcourt un pays étranger que par curiosité ou par désouvrement.

(18) Et tout le monde se remettait à rire, est-ce qu'il allait relever l'injure on est-ce qu'il allait l'accepter comme un défi et parce que, tout de même, fasciste, il l'avait été pendant dix ans par mépris et par haine pour des gens comme ceux-là ?

D’emblée, on remarque que la représentation schématique $X R Y$ évoquée ci-dessus, correspondant aux énoncés (6-18), appelle une lecture différente de celle qui concerne car, parce que et puisque (qui articulent, généralement, plus d'une seule proposition; ces conjonctions sont - rappelons-le - employées exclusivement dans l'expression de la "cause », voir supra), du fait que l'on a affaire à des phrases à verbe unique dont la séquence $R Y$ est composée d'une préposition $(R)$ et d'un régime $(Y)$ dont on peut établir une liste provisoirement intuitive (voir les critères infra, $\mathbb{\$} 1.3$.) : on a des $\mathrm{N}$ de sentiment (amour, jalousie, baine, etc.), de caractère humain (curiosité, vanité, etc.), de qualité (bumilité, bonté, modestie, etc.) d'états (nécessité, ennui, etc.).

Le schéma syntaxique correspondant à ces différentes occurrences en par présente un certain nombre de contraintes distributionnelles qui, pour nous, révèlent l'apport spécifique de la préposition par, son empreinte linguistique :

$$
\mathrm{N}_{0 \text { HUMAIN }}+\mathrm{V}_{\text {AGENTIF }}\left(\mathrm{N}_{1 \text { NON RESTREINT }}\right)+P A R \mathrm{~N}_{2}\left(+\Omega^{4}\right)
$$

Par conséquent, il est important d'étudier l'entour de par: la relation que la préposition entretient avec les différents constituants de la phrase conformément à ce que nous avons annoncé plus haut: la valeur "causale» est le résultat de l'interaction des différents éléments qui constituent l'énoncé.

\subsection{Les types de procès}

La première régularité observée au niveau des verbes dans les énoncés en par est qu'ils renvoient toujours à un procès dynamique, agentif ou à un état résultatif, mais jamais à un état permanent ou à une propriété intrinsèque. La situation énonce généralement une justification d'un changement constaté (quelque chose de nouveau), ce qui explique les inacceptabilités suivantes: *Les humains respirent par amour de la vie/par peur de mourir; *Paul grandit chaque jour de deux centimètres par jalousie de Pierre; * Les

\footnotetext{
${ }^{4}$ Le symbole $\boldsymbol{\Omega}$ de ce schéma indique que le $\mathrm{N}_{2}$ peut, dans certains cas, être modifié par un complément adnominal du type $N$ de $N$, mais l'observation du corpus a révélé que le $\mathrm{N} 2$ est rarement modifié (en termes de fréquence).
} 
bébés marchent à quatre pattes par fatigue/par sagesse, etc. où on n'a pas affaire à des changements, mais à des propriétés intrinsèques qui entrent dans la définition du sujet de la phrase (on note l'absence de volonté des sujets, comme le montre l'incompatibilité de ces phrases avec les verbes ou les adverbes suivants : décider/arrêter de (grandir), (grandir) volontairement, délibérément, intentionnellement, etc.); les procès possibles s'inscrivent forcément dans la durée et constituent une «rupture » par rapport à un état précédent, dont on fournit la cause ou le mobile dénoté par le GP en par: le locuteur énonce une justification de l'attitude ou de l'action assumée volontairement par le sujet.

\subsection{Les types de No}

Les différents $\mathrm{N}$ régis par la préposition par sont corrélés systématiquement à un nom humain $\left(\mathrm{N}_{0}\right)$, reconnaissable aux propriétés suivantes :-il est compatible avec l'interrogation en Qui ? (en position d'objet); il admet généralement sans problème la combinaison avec chez (chez Max/les Anglais/etc. versus *chez. (la table + la liberté + le chat, etc.); il peut être le sujet de certains verbes « intellectuels » ou de « sentiments »: Max (préfere + décide + choisit + prévoit + exige + croit + sait + prétend + juge + désire + rongit + doute + estime + etc.) ; il peut être l'objet de certains verbes «psychologiques »: Cela (ennuie + agace + énerve + tourmente, etc.) Max (cf. voir M. Gross, 1975 : 47-50).

Notons, cependant, qu'à la différence de par, certains marqueurs comme de peuvent se combiner avec un sujet non humain (désignant un animal ou une partie du corps humain) dans un rapport de «cause » : on peut avoir L'éléphant barrit de rage; Sa voix chevrote de peur (D. Leeman 1991 : 93), mais non *L'éléphant barrit par rage, ni *Sa voix chevrote par peur; il en va de même avec parce que: Une pierre tombe parce qu'elle est pesante (Stendhal). Du point de vue linguistique, la notion de « cause » avec par ne semble concerner que les êtres dotés de réflexion, de calcul, d'analyse et d'estimation. Il va de soi qu'une phrase comme Le chat mange la souris par mépris/cruauté; Les oiseaux chantent par amour/désceuvrement/ennui; Cette plante ne pousse que dans les pays chauds par peur de ne pas survivre/par sympathie/ par modestie; etc. sont des phrases que la logique humaine n'admet que par anthropomorphisme ${ }^{5}$; les animaux, du point de vue linguistique, suivent leurs instincts et ne raisonnent pas $^{6}$. Cette dernière constatation ne vient donc pas d'une simple réalité objective, mais d'une réalité langagière que justifient les différents critères linguistiques proposés ci-dessus.

\subsection{Les types de $\mathrm{N}_{2}$}

Il y a eu différentes tentatives de classement des noms comme amour, jalousie, haine, colère, impatience, fatigue, curiosité, etc. ${ }^{7}$; on leur attribue généralement l'appellation

5 Cf. S. Mejri (2003), pour la notion d'« anthropomorphisme» dans les séquences figées.

6 Sauf dans les dessins animés ou bien dans certains genres littéraires, comme les fables où les humains sont représentés allégoriquement par des non-humains.

7 On renvoie essentiellement à J.-C. Milner (1989), D. Leeman (1985, 1991, 1995, 1998), G. Kleiber (1981), N. Ruwet (1995) J.-C. Anscombre (1992, 1995, 2003), Y. Y. Mathieu (1994, 2000), A. BalibarMrabti (1995), et à H. Ounis (2006). 
«N psychologiques» (NPSY) avec un certain nombre de critères permettant de les identifier. Nous reprenons, dans ce qui suit, quelques-uns parmi les plus significatifs que l'on trouve dans J.-C. Anscombre (2003) ; l'auteur fait remarquer que l'une des propriétés principales des NPSY est qu'ils s'opposent aux noms agentifs: «A wellknown case is that of action nouns, and in some degree, psych-nouns are just the opposite of action nouns, as far as agentivity is concerned » (2003: 55). Les principaux critères proposés sont relatifs aux contraintes atteignant les déterminants et les distributions lexicales.

\subsection{Les contraintes sur les déterminants}

Généralement les NPSY ont en commun avec les noms «statiques» (par opposition aux noms «agentifs ») certaines contraintes au niveau du déterminant : ils admettent l'article partitif et ne sont pas naturels avec l'article défini (le/la), ni avec l'article indéfini (un/une), à moins que le $\mathrm{N}$ soit modifié par un adjectif, une relative ou bien s'insère dans une structure adnominale du type $N$ de $N$. De même, les $N_{P S Y}$ préfèrent le singulier et soit sont bizarres au pluriel (*admirations, *calmes, ${ }^{*}$ fatigues, ${ }^{*}$ confiances, etc.), soit changent de sens - ils prennent un sens événementiel ( $f$. amour versus amours; joie versus joies; chagrin versus chagrins; colère versus colères, etc.). Appliqué aux $\mathrm{N}$ du corpus cidessus, nous obtenons les résultats suivants :

- Le test du partitif (du/ de la)

Il y a (de l' + de la $+d u)$ (amour, bonté, cruauté, curiosité, décence, désouvrement, discrétion, ennui, baine, honnêteté, bumilité, jalousie, mépris, modestie, nécessité, peur, pitié, vanité) dans ce qu'il dit/dans l'air/dans ce qu'il fait/chez Max.

- Le test de l'article indéfini (le/la)

??Le l'la (amour, bonté, cruauté, curiosité, décence, déscouvrement, discrétion, ennui, baine, bonnêteté, bumilité, jalousie, mépris, modestie, nécessité, peur, pitié, vanité) caractérise Max.

- Le test de l'article indéfini un(e)

*Un(e) (amour, bonté, cruauté, curiosité, décence, désoeuvrement, discrétion, ennui, baine, honnêteté, humilité, jalousie, mépris, modestie, nécessité, peur, pitié, vanité) caractérise Max.

- Le test du pluriel

*(des/les) amours, bontés, cruautés, curiosités, décences, déscouvrements, discrétions, ennuis, baines, bonnêtetés, humilités, jalousies, mépris, modesties, nécessités, peurs, pitiés, vanités.

Ainsi, tous les $\mathrm{N}$ du GP par $N$ admettent la combinaison avec le partitif, ce qui confirme bien leur appartenance à la classe des $\mathrm{N}$ "statiques" par opposition à la classe des $\mathrm{N}$ "agentifs», comme construction, réparation, meurtre, etc. (cf. * Il y a de la construction, de la réparation, du meurtre, etc. dans ce qu'il fait/ dans ses projets). De même, ils 
sont incompatibles avec l'article défini et indéfini contrairement aux $\mathrm{N}$ agentifs ( $f$. La/ une construction; une/la réparation; un/le meurtre). En outre, ces $\mathrm{N}$ n'admettent pas le pluriel, en tous cas, en gardant le même sens; ceux qui l'admettent prennent automatiquement une certaine acception événementielle (ils deviennent «classifiants», selon les termes de J.-C. Milner (1989) ou «catégorématiques individuants» et même « syncatégorématiques », selon les termes de G. Kleiber (1981)). Ainsi, pour ne commenter que deux cas: cruauté (" caractère d'être cruel») versus cruautés (" manifestations effectives de ce caractère, par exemple, les souffrances infligées »); ennui ("sentiment désagréable dû au désœuvrement») et ennuis ("les problèmes »); etc. (voir, à ce propos, M. J. Jarrega, 2000 : 13-17). De toute façon, ces $\mathrm{N}$ ne sont jamais au pluriel quand ils sont construits avec par dans l'emploi dit «causal»: *Par amours cruautés/jalousies/ bontés/ennuis/etc.

L'incompatibilité des différents tests en rapport avec les déterminants définis, indéfinis ainsi qu'avec le test du pluriel est de nature à prédire la validité d'un autre critère proposé par J.-C. Anscombre (op. cit.) : les $\mathrm{N}_{\text {PSY }}$ ne se prêtent pas à la « distributivité » contrairement aux $\mathrm{N}$ « classifiants », comme voiture, arbre, chaise, etc. (cf. La voiture de Pierre et celle de Max sont dans le garage $=>$ Les voitures de Pierre et de Max sont dans le garage versus Le respect de l'bomme et celui de la nature vont de pair $=>$ *Les respects de l'bomme et de la nature vont de pair (ibid.). Ainsi, les NPSY - des N «qualifiant» - sont impossibles dans ce cas : La jalousie de Max et celle de Léa sont insupportables =>*Les jalousies de Max et de Léa sont insupportables ; L'amour de Max et l'amour de Léa sont bouleversants $=>$ * Les amours de Max et de Léa sont bouleversants 8 .

\subsection{Y a-t-il des cas où le $N_{2}$ admet l'insertion d'un déterminant dans le sens "causal» en par?}

Le recours au corpus de 1006 occurrences prises dans des situations discursives stabilisées a révélé que dans la valeur dite "causale », que l'on peut reconnaitre aux distributions lexicales données en $\ 1$ et ses ramifications, mais qui peut être identifiée aussi grâce au test classique de l'interrogation en Pourquoi ?/Pour quelle raison ?:

(7a) Pourquoi/Pour quelle raison me dépeignent-ils comme un monstre? - Par jalousie.

(10a) Pourquoi/Pour quelle raison n'a-t-il jamais dit de bien de qui que ce soit? - Par peur de se déprécier. Etc.

les énoncés dont le $N_{2}$ se construit avec un déterminant quel qu'il soit sont exclus. D'une part, l'insertion d'un déterminant n'est pas toujours possible :

(6a) L'une est unie par un lien mère-fils à l'autre, le fils en avant de la mère et la guidant par * $(u n e+s a+l a)$ nécessité plus que par (*un + son + ? l') amour.

\footnotetext{
8 Notons ici que le passage au pluriel ne devrait pas entraîner le genre féminin du $\mathrm{N}$ amour; en revanche, si le sens était «les histoires d'amour de Max et de Léa », la distributivité, dans ce cas, est acceptable, vu qu'il ne s'agit plus du sentiment d'amour proprement dit que l'on retrouve dans Par amour et, de toute façon, la phrase de départ ne peut pas être L'amour de Max et l'amour de Léa (...), mais Les amours de Max et les amours de Léa sont bouleversantes => Les amours de Max et de Léa sont bouleversantes.
} 
(7a) Ce sont les hommes, par (*une + leur + *la) jalousie, qui me dépeignent comme un monstre.

(8a) Les misérables paysans l'accablaient d'injures, lui lançaient, à son passage, des pierres, des cufs pourris, et de viles matières que par *(une $+m a+l a)$ décence je ne nomme pas.

(9a) Je l'ai fait tout autant par *(une + ma + la) vanité que par *(une + mon + l') bonnêteté.

(10a) Il n'a jamais dit de bien de qui que ce soit, par $\left({ }^{*} u n e+s a+l a\right)$ peur de se déprécier.

D'autre part, quand l'insertion d'un déterminant est possible, le sens bascule dans l'expression dite de «moyen » ou de «manière »; la question en Pourquoi ?/Pour quelle raison? n'est plus possible et c'est une tournure du type $A$ l'aide de quoi ?, Grâce à quoi ? ou Comment?/De quelle manière? qui prend sa place :

(19) Il l'a séduite par ruse $=>$

(19a) Pourquoi l'a-t-il séduite? - Par ruse («Il l'a séduite parce qu’il a une idée derrière la tête ; cela fait partie d'un stratagème »).

(20) Il les a indisposés par fatuité $=>$

(20a) Pourquoi les a-t-il indisposés? - Par fatuité («Il les a indisposés parce qu’il voulait se montrer supérieur $\approx$ «par vanité »).

Il en va autrement pour les exemples (21-22) où apparaît un déterminant; la valeur «causale » disparaît comme le montre le test de l'interrogation en Pourquoi ?

(21) Il l'a séduite par sa ruse $=>$

(21a) *Pourquoi l'a-t-il séduite? - Par sa ruse.

(22) Il les a indisposés par sa fatuité $=>$

(22a) *Pourquoi les a-t-il indisposés? - Par sa fatuité.

mais la question en Comment? (associée à la «manière » ou au «moyen ») est tout à fait possible :

(21b) Comment l'a-t-il séduite? - Par sa ruse ("Il l'a séduite grâce à l'usage de la ruse »).

(22b) Comment les a-t-il indisposés? - Par sa fatuité ("Sa fatuité lui a permis de les indisposer »).

De même, le passage du sens de « moyen »/ «manière » au sens « causal » (c'està-dire le cas inverse) en supprimant le déterminant n'est pas toujours possible :

(23) Il l'a séduite par sa vivacité d'esprit $=>$

(23a) */ ?Il l'a séduite par vivacité d'esprit.

(24) Il l'a séduite par son humour/intelligence $=>$

(24a) *Il l'a séduite par bumour/intelligence. 
Et dans beaucoup d'autres cas, la suppression du déterminant n'altère pas le sens global de l'énoncé de façon sensible, vu que l'on reste dans le même rapport discursif, le «moyen »/ la « manière » :

(25) On a acheminé la marchandise par (le + E) train.

Les deux types d'ajout s'opposent de trois manières :

- le $N_{2}$ dans les exemples (23-25) : bumour, intelligence, vivacité, train, etc. ne relève pas toujours de la classe des $\mathrm{N}$ caractérisée sous $\int 1.3$. (les NPSY) - points développé en $\int \mathrm{B}$;

- les $N_{2}$ ne connaissent pas les mêmes contraintes vis-à-vis de l'insertion d'un déterminant que ceux relevés dans l'emploi proprement «causal», par exemple en (6-18) ;

- l'insertion d'un déterminant possessif fait perdre à l'énoncé «causal » un trait essentiel pour qu'il soit reconnu en tant que tel ou pour convoquer un commentaire de type «causal», à savoir l'idée de «volonté» et celle de "finalité»: l'énoncé Il l'a séduite par sa ruse/son intelligence/son bumour/sa persévérance/son enthousiasme, etc. ne dit pas forcément que le sujet cherche à séduire la personne en question; cette dernière peut très bien se trouver séduite malgré elle par l'un des atouts du sujet (ruse, intelligence, bumour, persévérance, enthousiasme, etc.), alors qu'il ne fait aucun effort pour la séduire. De fait, les enchaînements possibles peuvent ne pas donner lieu à un commentaire de nature « causale ».

\subsection{Les distributions permises}

1.6.1. Les particularités des distributions lexicales

L'étude des distributions permises révèle que les $\mathrm{N}$ considérés appartiennent à diverses sous-classes des $\mathrm{N}_{\mathrm{PSY}}$, comme le montrent les possibilités et impossibilités combinatoires suivantes :

Eprouver/ressentir du/de la $N$ :

- amour, *bonté, *ruauté, *curiosité, *décence, *déscuvrement, *discrétion, ennui, haine, *honnêteté, *humilité, jalousie, mépris, *modestie, *nécessité, peur, pitié, *vanité.

Faire preuve de $N$ :

- *amour, bonté, *cruauté, curiosité, décence, *déscuvrement, discrétion, *ennui, *haine, bonnêteté, bumilité, *jalousie, *mépris, modestie, *nécessité, *peur, *pitié, *vanité.

Etre d'un(e) grand(e) $N$ :

- *amour, bonté, cruauté, curiosité, décence, *déscuvrement, discrétion, *ennui, baine, bonnêteté, humilité, jalousie, mépris, modestie, nécessité, *peur, ??pitié, vanité.

Etre dans un état de $N$ :

- *amour, *bonté, *cruauté, *curiosité, *décence, déscuvrement, *discrétion, ennui, *haine, *bonnêteté, *bumilité, ??jalousie, *mépris, *modestie, nécessité, peur, *pitié, *vanité.

Un sentiment de $N$ : 
Badreddine Hamma

- amour, *bonté, *cruauté, *curiosité, *décence, *déscuvrement, *discrétion, *ennui, haine, *bonnêteté, *bumilité, jalousie, ?mépris, *modestie, *nécessité, peur, pitié, *vanité

Ainsi a-t-on des sous-classes hétérogènes : on distingue des $\mathrm{N}$ renvoyant à des états permanents qui ne sont pas naturels avec des questions du type Depuis quand/combien de temps il est dans cet état ?/ Qu'est-ce qui s'est passé ? (cf. bonté, cruauté, curiosité, décence, discrétion, vanité, haine, bonnêteté, modestie, nécessitê); des $\mathrm{N}$ d'états passagers, déclenchés de façon extrinsèque et qui se combinent, globalement, avec des verbes comme provoquer, déclencher, etc. et qui admettent sans difficulté la question en Depuis quand/combien de temps il est dans cet état?/ Qu'est-ce qui s'est passé ? (cf. peur, désoeuvrement, ennui); on a aussi des $\mathrm{N}$ de «sentiment » (cf. amour, ennui, haine, jalousie, peur, pitiê) ; des $\mathrm{N}$ de « caractères humains » ou de « qualité » (cf. bonté, cruauté, décence, discrétion, honnêteté, bumilité, mépris, modestie, nécessité, pitié, vanitè). Le test en Faire preuve de est compatible avec les N (cf. bonté, décence, curiosité, discrétion, bonnêteté, bumilité, modestie) qui ont trait plutôt aux «qualités» (au sens de "caractère positif»), alors que la combinaison avec être d'un(e) grand(e) concerne les «caractères humains» de façon générale au-delà d'un jugement de valeur positif ou négatif. Par ailleurs, il existe un autre type de distributions affectant les $\mathrm{N}_{\text {PSY }}$ et qui permet de distinguer une autre propriété spécifique caractérisant l'emploi «causal» dans les énoncés en par: la combinaison avec un type particulier de prépositions.

\subsubsection{Distinction $\mathrm{N}_{P S Y}$ endogènes et $\mathrm{N}_{\text {PSY }}$ exogènes}

Dans la classe des $\mathrm{N}_{\mathrm{PSY}}$, J.-C. Anscombre (op. cit.) spécifie deux sous-classes : les $\mathrm{N}$ 《 endogènes » versus les $\mathrm{N}$ « exogènes »; leur distinction est rendue possible grâce au type des prépositions pouvant se construire avec ces $\mathrm{N}$ pour et envers (pour les « endogènes » et devant, à la vue de pour les « exogènes ». Reprenons les $\mathrm{N}$ du corpus considéré :

(26) L'amour de Max (pour + ?envers + *devant + *à la vue de) Léa

(27) La bonté de Max (pour + envers + *devant + *à la vue de) Léa

(28) La curiosité de Max (*pour + *envers + ?devant + *à la vue de) Léa

(29) L'ennui de Max (*pour + *envers + ?devant + *à la vue de) Léa

(30) La nécessité de Max (?pour + ?envers + *devant + *à la vue de) Léa

(31) La peur de Max (*pour + *envers + devant + à la vue de) Léa

(32) La discrétion de Max (*pour + *envers + *devant + *à la vue de) Léa

(33) Le mépris de Max (pour + envers + *devant + *à la vue de) Léa

(34) La vanité de Max (*pour + ?envers $+*$ devant + *à la vue de) Léa

(35) La baine de Max (pour + envers + *devant + *à la vue de) Léa

(36) L'bonnêteté de Max (*pour + envers + *devant + *à la vue de) Léa

(37) L'bumilité de Max (*pour + envers + devant + *à la vue de) Léa

(38) La jalousie de Max (?pour + envers + * devant + *à la vue de) Léa

(39) La modestie de Max (*pour +envers + *devant + *à la vue de) Léa

Nous constatons que les $\mathrm{N}_{\mathrm{PSY}}$ que par introduit se combinent tendanciellement avec les prépositions pour ou envers ou les deux, mais soit ils sont très bizarres avec à la vue de et devant, soit ils sont possibles sans empêcher le fait que la source du Npsy est 


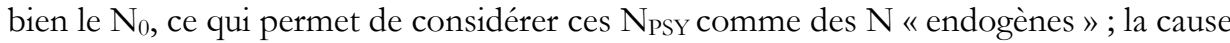
est ainsi reliée au sujet de la phrase $\left(\mathrm{N}_{0}\right.$ humain) qui constitue le "siège », le «lieu psychologique ». Cependant, les phrases (28-32) ne se combinent avec aucune des quatre prépositions; d'ailleurs, nous ne voyons pas quelle préposition pourrait apparaître dans ce contexte. Ce caractère «endogène » peut être confirmé par le fait que dans par $\mathrm{N}_{\mathrm{PSY}}$, c'est toujours le $\mathrm{N}_{0}$ (le sujet de la phrase) qui est concerné par le $\mathrm{N}$ psychologique comme le prouve le test avec le possessif; le déterminant sal son se rapporte toujours au $\mathrm{N}_{0}$ :

(a) Maxi est amoureux / jaloux de Léaj $=>$

(a') L'amour / La jalousie de Léaj $=>$

(a")*Saj jalousie/ *Sonj amour versus Saijalousie/ Soni amour).

(b) Maxi est haineux/ humble/ bon/ modeste/ envers Léaj $=>$

(b') La baine/ humilité/ bonté/ modestie $=>$

(b”) (*Saj haine/ humilité/ bonté/ modestie versus Sai haine/ humilité/ bonté/ modestie).

\subsection{Conclusion sur les distributions lexicales des NPSY de l'emploi «causal » de par}

A l'issue de l'examen des différentes contraintes linguistiques qui atteignent les $\mathrm{N}_{2}$ se construisant avec par dans le rapport de «cause », on peut dire qu'ils relèvent tous des NPSY, tout en appartenant à des sous-classes hétérogènes; ce qu'ils ont en commun, c'est le fait qu'ils sont statifs (= «non agentifs»), se rapportent tous à un sujet humain ( $\mathrm{N}_{0}$ HUMAIN) et se construisent tendanciellement avec par sans déterminant; le cas échéant, le NPSY est modifié par un adjectif, une relative ou entre dans une structure adnominale et, de façon générale, le GP (par $N$ ), dans ces occurrences, complète toujours des verbes d'action et jamais des verbes statiques; c'est le procès qui marque la rupture observée dans l'état de continuité établi par défaut et, partant, permet de parler d'une cause avec par en rapport avec le N NSY. Une deuxième caractéristique émerge des contraintes sur les prépositions pouvant se

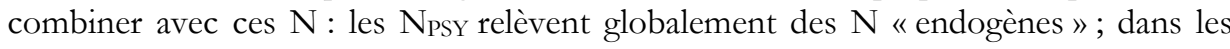
occurrences en par, ils informent sur le mobile à l'origine du procès dénoté par le verbe venant d'un sujet humain en tant que « source» ou « lieu psychologique ».

\section{Des propriétés distributionnelles au sens}

Dans ce qui précède, nous avons vu que le sens «causal » n’est pas exprimé par par de façon intrinsèque; il est à relier aux combinaisons lexicales (les distributions permises ou interdites par la langue) ainsi qu'au type de constructions syntaxiques mobilisé. Ce qui reste à faire maintenant, c'est d'étudier ce sens de plus près en établissant l'identité sémantique de la préposition par et en la comparant aux autres marqueurs pouvant entrer dans le même type de rapport discursif.

\section{1. Éléments heuristiques sur le sens de la préposition par}

L'un de nos principes théoriques - et qui prolonge les thèses saussuriennes et la linguistique descriptive, distributionnelle et transformationnelle, telle que considérée 
par Z. Harris - est que la langue est un système de signes autonome; les unités linguistiques ne s'y rapportent pas à un sens qui leur préexiste; celui-ci est inhérent au matériau verbal contenu dans l'énoncé et aux différentes interactions mises en jeu. Dans cette optique, chaque unité de la langue est dotée d'un signifié propre, une valeur invariante (qui ne se confond pas avec ce qu'elle évoque dans la réalité objective ou conceptuelle) qui, actualisé, donne à voir certains effets de sens («cause », " manière », « lieu », « instrument», «temps », etc.) sans que cet invariant sémantique soit éclipsé. Dans ce qui suit, nous rappelons succinctement l'invariant de par tel que nous l'avons établi à partir d'un travail empirique effectué sur un large corpus (B. Hamma, op. cit.).

Tout d'abord la préposition par se rattache à une construction élémentaire que nous pouvons déceler dans n'importe quel énoncé : par, son régime (par $R$ ) et un procès $(P)$ véhiculé généralement par le verbe et qui peut ne pas apparaittre en surface ; on peut le reconstituer à partir de paraphrases appropriées, notamment avec la notion de "phrase de base », telle que définie par Z. Harris ( $c f$. pour une présentation de ce principe harrissien, voir D. Leeman, 1998: 76-78); ainsi, dans l'emploi de par dit d'«agent» ( $c f$. L'Education sentimentale par Flaubert), on a un procès non donné explicitement, du type écrit, créé, conçu, etc. comme pourrait le confirmer le rapport établi de façon stéréotypique entre Flaubert ("romancier») et L'Education sentimentale («roman»). Ainsi, la relation (P) par $\mathrm{R}$ se présente comme une structure de base irréductible qui est sous-jacente à l'emploi de par dans toutes ses facettes. Cette régularité au niveau de la construction conduit à y discerner une régularité sémantique, une sorte d'invariance, conformément au «principe de naturalité » qui sous-tend notre démarche (cf. J.-C. Milner, 1989 : 307) : à une forme invariante correspond un sens invariant (de même qu'une différence de forme en appelle une autre sur le plan sémantique et vice versa).

Dans notre hypothèse, la séquence invariante $(P)$ par $R$ renferme un rapport sémantique particulier entre par $R$ et le procès $(P)$; on peut définir le $P$ dans les termes de R. Thom (1976) comme une « rupture » s'inscrivant dans une « continuité/stabilité relative» (état précédant la manifestation de cette "rupture») et qui renvoie, en l'occurrence, à l'élément nouveau qui appelle une « justification»; ainsi, dans l'énoncé (19) : Il l'a séduite par ruse, $P$ correspond à l'action volontaire du sujet ("séduire quelqu'un ») ; ce procès s'inscrit dans le temps et dans la durée (la séduction est bien un procès qui a eu lieu à un moment donné) et le syntagme prépositionnel par $\mathrm{R}$ (par ruse), correspond à la «justification »/à l'« explication » donnée par le locuteur de ce procès. Notons que, dans ce exemple, la finalité de la séduction telle que présentée par le locuteur, en l'absence de par $\mathrm{R}$, n'est pas évidente: par défaut, si l'on séduit quelqu'un, c'est parce que l'on est épris de lui, qu'il nous intéresse; mais, en l'occurrence, la justification rompt avec cette finalité : le sens est que la séduction fait partie d'un plan; on séduit pour obtenir quelque chose. De fait, par $R$ permet de donner une justification qui ne va pas de soi ; elle ne paraît pas évidente dans le cas où le locuteur n'apporterait pas cette précision. Sans Par N, l'interlocuteur établirait une justification par défaut (à partir de ce qu'il sait de la situation) dans un panel de justifications possibles; avec Par N, le locuteur ressent le besoin d'intervenir pour rectifier une raison sous-entendue ou paraissant aller de soi ou bien apporter une précision non évidente, une raison à laquelle on s'attend le moins. On peut résumer en deux points ces différentes notions heuristiques en rapport avec l'apport propre de par: 
Premier point : la justification en par est présentée par la langue comme contrastant avec d'autres : le par $\mathrm{R}$, dans ces énoncés, renferme dans la "phrase de base » qui lui correspond une opération de clivage (en C'est...qu...) dont on retrouve la trace dans les "phrases complexes» (celles réalisées dans le discours); celles-ci admettent le même enchaînement et le GP en par véhicule alors un certain « effet de contraste » (pour cette notion, voir D. Leeman, 1998 : 20-21) : Il est parti par pudeur signifie, en fait, que «si le sujet est parti, c'est uniquement parce qu'il est pudique et non pour une autre raison, par exemple, parce qu'il serait lâche, etc.». Le GP en par, dans cet énoncé, admet l'enchaînement en "...et non par R'» même en l'absence de l'opération de clivage en surface : Il est parti par pudeur et non par lâcheté. Le par R' (par lâchetê), dans ce cas, correspond à une tierce raison exclue et qui forme avec les $R$ réalisé dans l'énoncé considéré, dans la relation (par $\mathrm{R}$ ), un paradigme sélectionné par la préposition : « raisons d'agir corrélées au procès » (pudeur, jalousie, sympatbie, curiosité, peur, etc.).

Deuxième point : la «raison» réalisée dans un énoncé en par prend, du point de vue du locuteur, un certain aspect «non canonique/inhabituel», une situation «non standard»: selon les stéréotypes et les conventions (le «savoir partagé »), cette raison est présentée comme rompant avec ce à quoi on s'attendait. Cette hypothèse pour l'identité sémantique de par repose sur l'analyse de l'ensemble de ses emplois (B. Hamma, op. cit.), dont on ne donnera ici qu'un bref échantillon. Dans l'acception spatiale, on a par exemple remarqué que l'on ne dirait pas à un invité que l'on accueille chez soi Veuillez, entrer par la porte, s'il vous plaît mais seulement Vexillez, entrer s'ill vous plaît : la porte est le lieu normal pour pénétrer chez quelqu'un. En revanche, l'énoncé serait naturel en parlant d'un voleur : préciser qu'il est entré par la porte contredit les attentes (normalement, un voleur se cache, etc.) et convoque des implications a priori non évidentes (par exemple: si le voleur est entré par la porte, c'est que celle-ci était ouverte, ce qu'elle n'aurait pas dû être en l'absence de l'habitant légitime de l'endroit). De même, par terre précise une localisation qui n'est pas celle que l'on établit par défaut pour Il est assis, et dans Tomber par terre, c'est l'ensemble du prédicat qui affecte au sujet une position anormale dans Le stylo est tombé par terre (si l'on tombe, c'est normalement par terre, certes, mais c'est le résultat qui décrit une situation anormale : le stylo se retrouve par terre alors qu'on l'attendrait sur le bureau ou dans le tiroir). Pareillement, la spécification par la main dans Prendre un enfant par la main construit une situation différente de ce que dit Prendre un enfant (qui appelle par défaut l'interprétation "prendre un enfant dans ses bras»).

Les emplois temporels tels qu'illustrés par le Grand Larousse de la langue française se prêtent à la même analyse sémantique : le nom y est nécessairement précisé (par ces temps de misère, par les temps qui courent, par les époques troublées comme celles que nous traversons) par un modifieur introduisant un point de vue (ce qui n'est pas uniquement partagé et s'oppose à une autre vision des choses); dans On célébre cette fête avec moins d'éclat que par le passé, l'implication est que l'on pourrait s'attendre au contraire que la fête en question soit célébrée avec un éclat particulier à l'époque actuelle. De même, Il faut du courage pour se baigner par un froid pareil s'oppose à une doxa sous-jacente « on ne se baigne pas lorsqu'il fait très froid».

Préciser par lui-même dans Cet incident est par lui-même sans gravité est également destiné à prévenir une conclusion erronée chez l'interlocuteur : sans gravité et incident 
ont la même orientation argumentative et conduisent à la conclusion que l'on ne doit pas s'inquiéter; par lui-même inverse l'orientation, conduisant à l'inférence que l'on doit s'inquiéter des conséquences qu'il a par ailleurs. Une locution telle que par moments ne s'associe pas non plus à n'importe quelle situation supposant la répétition d'une action ou d'un événement : ainsi, dire d'un étudiant Il va en cours par moments ou Il écoute le professeur par moments ne signifie pas uniquement qu'il va en cours ou écoute le professeur autant de fois qu'il est nécessaire ou qu'on s'y attend, mais y ajoute l'idée que cette fréquentation des cours ou cette attention à leur contenu n'est pas conforme à ce à quoi on peut généralement penser a priori.

Cet aspect «inhabituel» et «non attendu» apparait dans tous les emplois dits de «cause» de par. Cela se confirme par le fait que le régime de par n'admet généralement qu'un seul type de modification dont le sens est de détacher ce $R$ des autres comme une entité singulière, avec des adjectifs du type pur, simple, etc. :

(40) Et encore par simple curiosité, sans que jen sois spécialement ému.

(41) Je pourrais prendre le coup de téléphone de Jules comme un signe du destin qui m'empêcherait d'envoyer la lettre, mais mon ressentiment à l'encontre de Marine est tel que, par pure méchanceté, je descends immédiatement lui poster cette lettre validée par la rumeur, j'aurai beau jeu ensuite de prétendre que le coup de fil de Jules était survenu juste.

(42) Non seulement on ne peut plus y respirer mais si l'on baisse les yeux, et il le faut bien, par élémentaire prudence, le cour se soulève de dégoût (Frantext).

Dans le paragraphe suivant, nous allons voir comment nos hypothèses en rapport avec le sens instructionnel de par apparaissent dans l'expression de la «cause » en comparant cette préposition avec d'autres marqueurs, principalement, de, en raison de et à cause de proposés par les ouvrages de référence comme synonymes de par.

\subsection{Le sens de par $\mathrm{N}_{\mathrm{PSY}}$ opposé à celui de de $\mathrm{N}_{\mathrm{PSY}}$}

Si certains GP, comme en de NPSY admettent des verbes comme rayonner, resplendir, rougir, pleurer, trépigner, bouillir, burler, griller, bouillonner, péter, trembler, chevroter (de baine + de joie + d'émotion + d'orgueil, etc.), le GP par NPSY, lui, ne semble pas s'accommoder de ce genre de verbes qui dénotent un état d'émotion non contrôlé en rapport avec les parties du corps (cf. D. Leeman, 1991 : 80-81) :

(43) *(Rayonner, resplendir, rougir, pleurer, trépigner, bouillir, burler, griller, bouillonner, trembler, chevroter) par (amour + haine + joie + émotion, etc.).

Nous avons démontré supra que le $\mathrm{N}_{0}$ qui se combine avec par, dans ce type d'occurrences, est obligatoirement un sujet humain qui agit de telle façon qu'une certaine finalité/un résultat est obtenu, ce qui explique les inacceptabilités suivantes:

*Max (frémit + tremble + cheurote + trépide) par peur/honte/pudeur/ réserve/ timidité.

Par présuppose que le sujet soit conscient de ce qu'il fait et le procès qu'il prend en charge est impérativement un verbe d'action ou présenté comme conduisant à un 
état résultatif nouveau (ce que nous avons défini comme « rupture ») ; a contrario, le GP en de $N$ n'admet pas les verbes agentifs :

(45) *Il travaille de plaisir ; *Il est parti de honte ; *Il n'a rien dit de pudeur ; etc.

Le sens de la cause avec de NPSY est de l'ordre d'une « rupture » observée, déjà là, mais qui est incontrôlable; nous retrouvons, de fait, la valeur de l'« origine » exprimée par de dans Le cabier de Paul; Finir de manger; Aller de Paris à Marseille ; L'avion de Berlin; De bouche à oreille; etc. Or, ce type de procès (verbes dynamiques/d'activité s'opposant aux verbes statiques et aux verbes affectant les parties du corps) donne des phrases acceptables avec par associé aux mêmes Npsy, ci-dessus (peur/ bonte/ pudeur/ réserve/ timidité) :

(46) Réponse simple et pure: il ne m'en a jamais parlé de ce gentil frère, par pudeur.

(47) Par amour, papa ne sait rien dissimuler à maman.

(48) Il se sentait joué, mais, par peur, ne voulait rien laisser paraître.

(49) Personnellement, il s'en était presque toujours gardé, au début par timidité et par peur de sa maladresse, ensuite par habitude et par souci de préserver son indépendance.

(50) Ce n'était qu'une fillette souffrant d'un lupus qui se présentait toujours voilée de noir, non par bonte de son mal, mais parce que Zénon avait remarqué que la lumière en accroissait les ravages.

(51) Quand j'inventai, pour ma porte étroite, le nom d'Alissa, ce ne fut point par préciosité mais par réserve.

Ce caractère agentif du sujet et du procès peut se voir à travers le test de l'interrogation :

(52) Pourquoi agit-il ainsi? Qu'est-ce qui l'a poussé à agir ainsi? Pour quelle raison a-t-il fait cela?, la réponse pouvant être Par amour/bonte/réserve/pudeur/timidité!, ce qui n'est pas le cas avec de $N$ :

(53) *Pourquoi trépigne/frémit/tremble-t-il? - De panique/ honte/peur, etc.

Ces différentes hypothèses sont également confirmées par le test de la commutation qui montre l'incompatibilité de leur interchangeabilité :

(54) Ils n'en distinguent rien, (par + * de) haine de l'obscurité.

(55) Chaque matin, Lucien et moi partions ensemble et, (par $+{ }^{*}$ de) prudence, je ne le quittais qu'à la porte de son école.

(56) Un matin, (par $+{ }^{*}$ de) curiosité, je me rendis derrière les Quinconces.

(57) Cette réaliste si fine, égarée dans une famille de spiritualistes grossiers, se fit voltairienne (par + *de) défi sans avoir lu Voltaire. 
Quelqu'un a frappé à la porte et, (par + *de) peur, elle n'a pas ouvert: le visiteur est reparti, par sa faute.

(59) Dans un autre journal, un certain Capo de Feuillide compara le roman à "un livre de $M$. Sade »dont il se refusait, (par + *de) pudeur, à citer même le titre.

(60) (Par + *D') amour de l'amour, George Sand était incapable de rester longtemps une femme sans homme.

(61) Il avait le visage poupin d'un chérubin qu'une méchante fée, (par + *de) vengeance ou (par + * de) jalousie, aurait changé en chanoine.

Toutefois, quand leur commutation est possible, par exemple dans siffloter $($ par + de) plaisir, cela ne correspond pas au même sens. De même, on aurait travailler par plaisir, mais non *travailler de plaisir et à l'inverse rougir de plaisir mais non * rougir par plaisir. De ce fait, nous pourrions dire que :

- $\quad$ travailler est un verbe d'action, qui suppose un sujet agentif ; on peut dire par exemple Il fait semblant de travailler, Il décide de travailler, Il travaille efficacement, et l'on peut enjoindre à quelqu'un : Travaille!

- en revanche, rougir est un verbe d'état, dont le sujet est un "patient»; sauf par plaisanterie, on ne peut pas dire Il fait semblant de rougir, Il décide de rougir, Il rongit efficacement ni enjoindre Rougis!

Donc par indiquerait la cause en relation avec un procès agentif et de avec un procès non agentif : le sens de rougir de plaisir est que le plaisir que l'on ressent fait rougir sans qu'on le veuille ou qu'on le contrôle, alors que le sens de travailler par plaisir est que l'on travaille parce qu'on le décide et que l'on y trouve du plaisir, donc la relation est en fait inverse ; d'une certaine manière, il y a une idée de « finalité »/«but » avec par puisque travailler par plaisir suppose que l'on travaille pour éprouver du plaisir (à travailler), que le travail est la source de ce plaisir. Ainsi, ce qui opposerait siffloter de/par plaisir, c'est que, globalement, dans le premier cas la personne ressent du contentement, ce qui la fait siffloter sans que cela soit un acte délibéré ou conscient, tandis que dans le deuxième cas, la personne décide de siffloter parce qu'elle aime cela. Avec de, le plaisir est déjà dans la personne, c'est lui qui cause le déclenchement du plaisir; en revanche, par suppose une extériorité du sentiment (qui est visé, qu'on veut atteindre). C'est peut-être ainsi qu'on peut expliquer Rougir par pudeur (opposé à de pudeur) : par serait la cause alléguée par celui qui parle (je dis, moi, observateur extérieur, que c'est la pudeur qui le/la fait rougir), de serait la cause avérée contenue dans celui/celle qui rougit.

\section{Conclusion générale}

Il ressort de ce qui précède qu'il n'est pas pertinent de parler de "préposition causale » à propos de par. Nous avons vu que cette valeur - c'est d'ailleurs le cas des autres valeurs («lieu», «temps», «moyen», etc.) - est le résultat de l'interaction de chacun des items pouvant apparaitre dans un énoncé véhiculant ce type de rapport 
discursif et non seulement du morphème en soi : le procès, par exemple, a un rôle très déterminant. Dans l'emploi «causal», il constitue une «rupture» observée, ou une "discontinuité »/un "conflit», pour reprendre les termes de R. Thom, qui se démarque de l'état de "continuité » établi par défaut, et dont on éprouve le besoin de préciser la «cause» qui a conduit à un tel «conflit», ce que prend en charge le segment Par R. Nous avons vu également que l'étiquette "cause», elle-même, est problématique, dans la mesure où elle ne prévient pas des cas où la commutation est impossible avec les marqueurs concurrents ; ainsi, par permet de voir la cause sous un angle singulier, par opposition à d'autres prépositions comme $d e$ : par, entre autres propriétés, se rattache forcément à un sujet humain et non les autres marqueurs. De plus, la cause dans les énoncés en par s'inscrit dans un scénario de cause «non canonique », "inhabituelle», contrairement à celle en de, par exemple, qui informe d'une cause plutôt évidente et avérée. Ainsi, notre étude aura montré que la préposition par présente la valeur « causale » sous un angle particulier en l'imprégnant de sa propre couleur de sens, que l'on peut déceler comme invariant sémantique dans tous les emplois de la préposition.

\section{RÉFÉRENCE BIBLIOGRAPHIQUE}

Anscombre, J.-C., 1992, "Quand on fait du sentiment ; réflexions (presque) spontanées sur la nature des noms psychologiques », Hommages à Nicolas Ruwet, Ghent, Communication et Cognition : 139-154.

Anscombre, J.-C., 1995, «Morphologie et représentation événementielle : le cas des noms de sentiment et d'attitude », Langue française 105, Paris, Larousse : 40-54.

Anscombre, J.-C., 2003, "Psych-nouns in French: semantics and object classes », Language Research Special Issue, Seoul National University, Seoul, Korea : 55-76.

Balibar-Mrabti, A., 1995, «Grammaire des sentiments » Langue française 105, Paris, Larousse.

CADIOT, P., 1997, Les prépositions abstraites en français, Paris, Armand Colin.

FRANTEXT : http://www.frantext.fr/Dendien/scripts/categ/displayp.exe

Gross, M., 1975, Méthodes en syntaxe. Régime des constructions complétives, Paris, Hermann.

HAMMA, B., 2005, L'invariant sémantique de la préposition par à travers les distributions syntaxiques et lexicales, Thèse de doctorat, Université de Nanterre.

Hamma, B., 2005a, "Travail de corpus et modélisation des données: le cas des

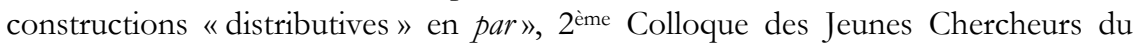
Laboratoire MoDyCo, Paris X - Nanterre.

HAMON, S., 2005, La phrase double causale : propriétés syntaxiques et interprétations sémantiques, Thèse de doctorat, Université de Nanterre. 
Harris, Z., 1976, Notes du cours de syntaxe, M. Gross [éd.], Paris, le Seuil.

JARREGA, M. J., 2000, Le rôle du pluriel dans la construction du sens des syntagmes nominaux en français contemporain, Thèse de doctorat, Université de Nanterre.

KLEIBER, G., 1981, Problèmes de référence : descriptions définies et noms propres, Paris, Klincksieck.

KWON-PAK S.-N., 1997, Les prépositions spatiales: sur quelques emplois de PAR, Thèse de doctorat, Université de Strasbourg.

LeEman, D., 1985, «Tentative de caractérisation d'un complément circonstanciel : Dans mon affolement, je lâchai mon panier de cerises », Linx 12 : 97-146.

LEEMAN, D., 1990, "Sur les compléments circonstanciels », Langue française 86, Paris, Larousse : 3-10.

LEEMAN, D., 1991, "Hurler de rage, rayonner de bonheur: Remarques sur une construction en de », Langue française 91, Paris, Larousse : 80-101.

LeEman, D., 1995, "Pourquoi peut-on dire Max est en colère mais non * Max est en peur? Hypothèses sur la construction être en $N »$, Langue française 105, Paris, Larousse : 5569.

Leeman, D., 1998, Les circonstants en question(s), Paris, Kimé.

MathiEU, Y.-Y., 1994, «Verbes psychologiques et interprétation sémantique », Langue française 105, Paris, Larousse : 98-106.

MATHIEU, Y.-Y., 2000, Les verbes de sentiment. De l'analyse au traitement automatique. CNRS, Paris.

MejRI, S., 2003, «La stéréotypie du corps dans la phraséologie. Approche contrastive », in Burger Harald, Hacki-Buhofer Annelies, Greciano Gertrud, (éds), Flut von TextenVielfalt der Kulturen, Phraseologie und Parömiologie, Band 14 : 203-217.

MiLnER, J.-C., 1989, Introduction à une science du langage, Paris, Seuil.

NORMAND, C., 2002, «la sorcière, car c'était elle. Remarques sur des réponses anticipantes », Colloque de Chambéry en hommage à Michèle Perret, (à paraître).

Ounis, H. ET D. LEEMAN, 2003, «Coup de foudre: métaphore ou signifié dans la langue »?", Chercher les passages avec Daniel Delas, Pari, Harmattan.

OunIS, H., 2006, Lexique et métaphore : le champ de l'amour, Thèse de doctorat, Université de Nanterre.

RUWET N., 1995, «Les verbes de sentiment forment-ils une classe distincte dans la grammaire?", in Tendances récentes en linguistique française et générale, volume dédié à David Gaatone, Linguisticae Investigationes Supplementa, Amsterdam/Philadelphia, Offprint. 